\title{
Audit to Assess the Implementation of Early First Feeding in Newborns after Caesarean Delivery
}

\section{Ruby Jose ${ }^{*}$, Pushplata Kumari, Reeta Vijayaselvi and Manisha Madhai Beck}

Head of Obstetrics and Gynaecology Unit 4 Christian Medical College and Hospital, Vellore, India

*Corresponding author: Ruby Jose, Professor, Head of Obstetrics and Gynaecology Unit 4 Christian Medical College and Hospital, Vellore, India, E-mail: rubyjose1@gmail.com

Received: July 15, 2015; Accepted: July 31, 2015; Published: August 10, 2015

Copyright: (c) 2015 Jose R. This is an open-access article distributed under the terms of the Creative Commons Attribution License, which permits unrestricted use, distribution, and reproduction in any medium, provided the original author and source are credited.

\section{Background \\ Period}

Current National and International [1-3] guidelines strongly recommend initiating breastfeeding $(\mathrm{BF})$ within one hour of birth. The advantages of initiating BF early are establishment of successful lactation and giving first milk or colostrum which is advantageous to the baby. In addition, a study from Ghana showed that by initiating BF within one hour of birth, $22.3 \%$ of all neonatal deaths could be prevented [4]. Another epidemiological study had documented a causal association between early BF and infection related mortality in the NB [5].

Whereas initiation of BF is carried out by and large, within an hour after vaginal birth, it is not the same after a Caesarian Delivery (CD). $\mathrm{BF}$ may be delayed after $\mathrm{CD}$ due to various reasons. One of the often quoted reasons is that after LSCS, women are not conscious within an hour after general anaesthesia (GA), or, even if regional anaesthesia was employed, they were over sedated and unable to breastfeed effectively. Most CDs are carried out under regional anesthesia. GA is used only for specific situations where regional anethesia is contraindicated, as with low platelet count in pre eclampsia or with heavy bleeding being anticipated as in major degree Placenta previa. BF should be commenced within the hour with the same fervour in post LSCS women as in women after vaginal delivery.

\section{Audit Scope}

Early initiation of BF can decrease infection related neonatal mortality and establish lactation successfully. This audit was done to assess BF practice in post LSCS mothers in this tertiary level teaching hospital with scope for improving the practice.

\section{Aim}

To assess the implementation of early first BF in the NB after CD in a tertiary level, teaching hospital of South India.

\section{Objective}

To determine the time between $\mathrm{CD}$ and the first $\mathrm{BF}$ in eligible newborns in the maternity wards

\section{Material and methods}

\section{Setting}

Maternity ward, Christian Medical College and Hospital, Vellore

\section{Inclusion criteria}

1. NB born by CD

2. Both mother and NB shifted to postnatal ward after delivery.

\section{Exclusion criteria}

1. NB shifted to nursery for neonatal complication.

2. Mother with any morbidity that requires close monitoring in the ICU (severe pre eclampsia/ eclampsia, post-partum bleeding)

\section{Mothers who had stillbirths}

All eligible babies, were Included in this audit. The time of first BF after birth was obtained from the nurse's record and cross checked with the mother.

\section{Results}

General Characteristics: The total number of CDs during the 5 day period was 61 and the number of babies shifted to the mothers side was $49(80.23 \%)$. Twelve of the NB was not included in the audit as they were not shifted to the mother side because of a neonatal complication. None of the mothers included in the audit had any exclusion criteria. The mean birthweight was $2909.4 \mathrm{~g}, 45$ of them were term and 4 were preterm. There were more male NB than females (20 female- $40.8 \%$ and 29 males $-59.2 \%$ ) (Figure 1 ).

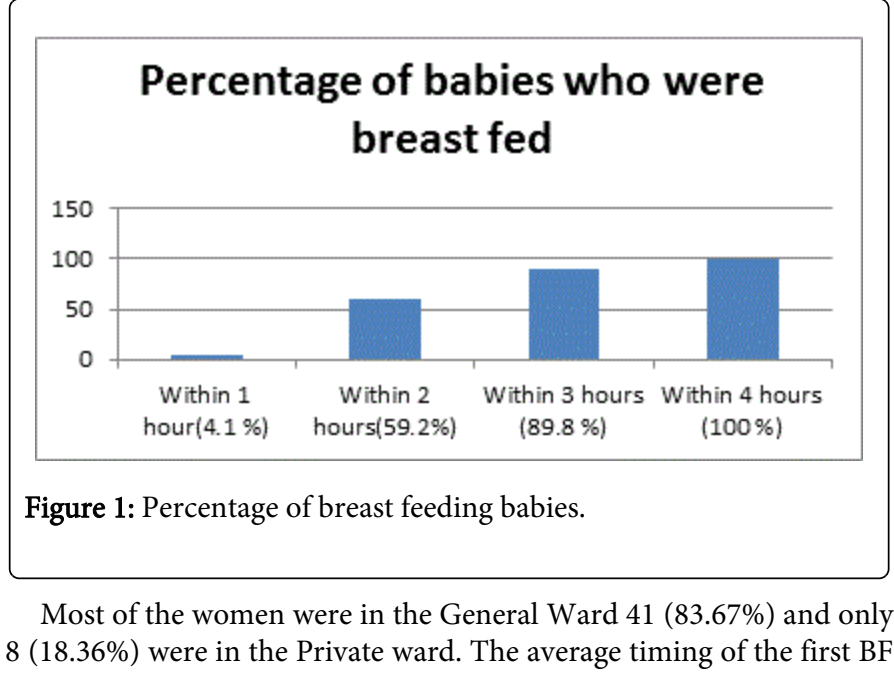


Citation: Jose R, Kumari P, Vijayaselvi R, Beck MM (2015) Audit to Assess the Implementation of Early First Feeding in Newborns after Caesarean Delivery . J Neonatal Biol 2015 4: 185. doi:10.4172/2167-0897.1000185

Page 2 of 2

following $\mathrm{CD}$ was $123.4 \pm 36.4$ minutes, the range being 58 to 225 minutes.

Only $4.1 \%$ commenced first $\mathrm{BF}$ within the first hour. Within the first 2 hours, $59 \%$ had first BF. While it took 4 hours to for $100 \%$ first $\mathrm{BF}, 89.8 \%$ had first BF by 3 hours after CD.

\section{Discussion}

In this audit we have found that only $4.1 \%$ of mothers who had LSCS had started breast feeding during the first hour of life. As per publication by The Ministry of Women and Child Development in $2004,15.8 \%$ of mother had initiated BF within 1 hour in India [2]. In a study conducted by Radhakrishnan and Balamuruga in rural women in Tamil Nadu, $60.5 \%$ of women had started breastfeeding within half an hour after birth [6]. According to the National Family and Health Survey - 3, 23.4\% of mother had initiated breastfeeding within $1 \mathrm{~h}$ after birth [7]. In all the above quoted studies, all births were included. None of the studies had categorised CD, where first BF is even further delayed.

The current data showed that all mothers had started breastfeeding within 4 hours of life and approximately $90 \%$ within 3 hours. This is good when compared with the study conducted on rural women in Tamil Nadu [6] where only $72 \%$ of the mothers had initiated BF within 4 hours. In another study by Ranjana et al., the percentage of mothers who had initiated BF within the first six hour was only $70 \%$ [8].

\section{Conclusion}

Following $\mathrm{CD}$, all the mothers had initiated $\mathrm{BF}$ within the first four hours of birth, but only $4.1 \%$ of mothers did it in the first hour, as is recommended. This important aspect has been neglected by both the Obstetricians and the Pediatricians in this teaching hospital. The reasons for this could be that the Faculty are caught up with looking after High Risk Mothers and their NB. Another reason that could be attributed is that the subject of BF belongs to both the Obstetrician and the Neonatologist. In the long run, this will eventually lead to more neonates dying due to discontinuation of $\mathrm{BF}$ and neonatal infections as well.
The introduction of "care managers", as a bridge between the specialist and the patient may result in improvement of clinical parameters and better adherence to BF, just as the study by Ciccone et al. [9] demonstrated that the management of the diabetic patient by the introduction of a specialized nurse determines a better control of hyperglycemia, hypertension, and hyperlipidemia.

\section{Recommendations}

Postoperative ward nurses and doctors should have special refresher sessions to explain the importance of initiating early breastfeeding in new mothers.

These sessions should be repeated at regular intervals and audits should be repeated to monitor the improvement.

\section{References}

1. (2009) World Health Organization. What is the recommended food for children in their very early years?

2. Infant and Young Child Feeding Chapter, Indian Academy of Pediatrics, Rajeshwari K, Bang A, Chaturvedi P, et al. (2010) Infant and young child feeding guidelines: 2010. Indian Pediatr 47: 995-1004

3. Breastfeeding promoting network of India.

4. Edmond KM, Zandoh C, Quigley MA, Amenga-Etego S, Owusu-Agyei S, et al. (2006) Delayed breastfeeding initiation increases risk of neonatal mortality. Pediatrics 117: e380-386.

5. Edmond KM, Kirkwood BR, Amenga-Etego S, Owusu-Agyei S, Hurt LS (2007) Effect of early infant feeding practices on infection-specific neonatal mortality: an investigation of the causal links with observational data from rural Ghana. Am J Clin Nutr 86: 1126-1131.

6. Radhakrishana and Balamuruga (2012) Prevalance of exclusive breastfeeding practices among rural women in Tamil Nadu. International journal of Health and Allied sciences 1: 64-67.

7. National Family Health Survey (NFHS-3). Factsheets 2005-2006.

8. Ranjana F, Lakshminarayana J, Ramnath T, Singh Madhu B (2002) Health and nutritional status infant feeding practices of working women in Jodhpur city. Ann Arid Zone 41: 183-189.

9. Ciccone MM (2010) Feasibility and effectiveness of a disease and care management model in the primary health care system for patients with heart failure and diabetes. Vasc Health Risk Manag 6: 297-305. 